\title{
Chapter 14 \\ Future Potential of Herbal-Based \\ Medicinal Treatment for Management of Asthma
}

Plant-based medicine, sometimes called as phytomedicine has been successful in effective treatment of asthma (Aher et al. 2009). The market for phytomedicine has always been huge, where close to three-fourth of the prescribed and over-thecounter drugs are prepared from natural and plant-based resources (Shakarami et al. 2019). Respiratory ailments have long been an area of active research and several plant-based formulations have provided complete, successful, long-term symptomatic relief. In summary, the future of phytomedicine towards the treatment of asthma holds immense potential. 